\title{
Evaluating genome-wide DNA methylation changes in mice by Methylation Specific Digital Karyotyping
}

\author{
Kathy Boon*1, John K Tomfohr1, Nathaniel W Bailey ${ }^{1}$, Stavros Garantziotis ${ }^{1}$, \\ Zhuowei Li ${ }^{2}$, David M Brass ${ }^{1,2}$, Shuichiro Maruoka1, John W Hollingsworth ${ }^{2}$ \\ and David A Schwartz 1,3,4
}

\begin{abstract}
Address: ${ }^{1}$ National Heart Lung and Blood Institute/National Institute of Environmental Health Sciences, Research Triangle Park, NC 27709, USA ${ }^{2}$ Duke University Medical Center, Durham, NC 27710, USA, ${ }^{3}$ National Jewish Health, Denver, CO 80206, USA and ${ }^{4}$ University of Colorado Health Sciences Center, Denver, CO 80206, USA

Email: Kathy Boon* - boonc@mail.nih.gov; John K Tomfohr - tomfohr@gmail.com; Nathaniel W Bailey - getnatbailey@gmail.com; Stavros Garantziotis - garantziotis@niehs.nih.gov; Zhuowei Li - zhuowei.li@duke.edu; David M Brass - david.brass@duke.edu; Shuichiro Maruoka - maruokas@niehs.nih.gov; John W Hollingsworth - holli017@mc.duke.edu; David A Schwartz - schwartzd@njc.org

* Corresponding author
\end{abstract}

Published: II December 2008

BMC Genomics 2008, 9:598 doi:10.1/86/147|-2164-9-598

This article is available from: http://www.biomedcentral.com/I47I-2/64/9/598

(c) 2008 Boon et al; licensee BioMed Central Ltd.

This is an Open Access article distributed under the terms of the Creative Commons Attribution License (http://creativecommons.org/licenses/by/2.0), which permits unrestricted use, distribution, and reproduction in any medium, provided the original work is properly cited.
Received: 7 May 2008

Accepted: II December 2008

\begin{abstract}
Background: The study of genome-wide DNA methylation changes has become more accessible with the development of various array-based technologies though when studying species other than human the choice of applications are limited and not always within reach. In this study, we adapted and tested the applicability of Methylation Specific Digital Karyotyping (MSDK), a non-array based method, for the prospective analysis of epigenetic changes after perinatal nutritional modifications in a mouse model of allergic airway disease. MSDK is a sequenced based method that allows a comprehensive and unbiased methylation profiling. The method generates 21 base pairs long sequence tags derived from specific locations in the genome. The resulting tag frequencies determine in a quantitative manner the methylation level of the corresponding loci.
\end{abstract}

Results: Genomic DNA from whole lung was isolated and subjected to MSDK analysis using the methylation-sensitive enzyme Not I as the mapping enzyme and Nla III as the fragmenting enzyme. In a pair wise comparison of the generated mouse MSDK libraries we identified I58 loci that are significantly differentially methylated $(P$-value $=0.05)$ after perinatal dietary changes in our mouse model. Quantitative methylation specific PCR and sequence analysis of bisulfate modified genomic DNA confirmed changes in methylation at specific loci. Differences in genomic MSDK tag counts for a selected set of genes, correlated well with changes in transcription levels as measured by realtime PCR. Furthermore serial analysis of gene expression profiling demonstrated a dramatic difference in expressed transcripts in mice exposed to perinatal nutritional changes.

Conclusion: The genome-wide methylation survey applied in this study allowed for an unbiased methylation profiling revealing subtle changes in DNA methylation in mice maternally exposed to dietary changes in methyl-donor content. The MSDK method is applicable for mouse models of complex human diseases in a mixed cell population and might be a valuable technology to determine whether environmental exposures can lead to epigenetic changes. 


\section{Background}

In eukaryotes DNA methylation occurs on cytosine residues of CpG dinucleotides. CpG islands, short genomic regions with a high frequency of $\mathrm{CpG}$ dinucleotides, are typically common near transcription start sites, and may be associated with promoter regions. These regions are not generally methylated in contrast to highly repetitive elements in the genome. DNA methylation can directly affect transcription by inhibiting binding of specific transcription factors and/or enhancing necessary chromatin remodeling for gene silencing. This process is required for normal embryonic development, imprinting, and X chromosome inactivation, and plays an important role in the normal functioning of an organism. Increasing numbers of studies are emphasizing the role of DNA methylation in human diseases [1-3]. Only until recently it has become clear that nutritional components can also affect epigenetic mechanisms like DNA methylation and can lead to long term phenotypic changes $[4,5]$.

The study of DNA Methylation has become more accessible by recent development of various technologies [6]. The choice of methodology is highly dependent of the goal of the study, genome of interest and available resources. Most commercially available micro-array platforms are developed for restriction enzyme and affinity based assays like the short oligonucleotide arrays (Affymetrix), tiling arrays (NimbleGen) and CpG Island/ promoter arrays (Agilent) [6]. Like in other micro-array assays intensity changes are measured instead of actual levels of methylation and are subject for many sources of variation like array-lot variability and washing conditions. Whether the hybridization intensity will be proportional to the level of methylation is still uncertain and could be platform dependent. While these approaches are able to evaluate methylation changes throughout the entire genome, they remain expensive and are not generally accessible. Methylation Specific Digital Karyotyping (MSDK) is a non-micro array, genome-wide methylation analysis approach that relies on cleavage of genomic DNA with a methylation sensitive restriction enzyme. The concept of this approach is similar to serial analysis of gene expression (SAGE) and is based on the following principles: 21 bp sequence tags are derived from specific locations within the genome and can be directly matched to their corresponding loci, and concatenation of the sequence tags allows the serial analysis of multiple tags by high throughput sequencing $[7,8]$. The resulting genomic tag frequencies determine in a quantitative manner the methylation level of the corresponding loci. In this report, we present a modification and application of MSDK, for the study of methylated loci throughout the mouse genome prenatally exposed to nutritional variations.

\section{Results and discussion}

\section{Genome-wide DNA methylation analysis}

In an effort to uncover candidate genes directly affected by DNA methylation in a mouse model of perinatal nutritional modification and allergic airway disease, we applied MSDK, a comprehensive, quantitative and unbiased genome-wide method that offers accurate information on the genomic position of differentially methylated loci. The MSDK method has been previously developed for the analysis of the human genome using the restriction enzyme Asc I as the mapping enzyme. The Asc I recognition site is preferentially found in CpG islands associated with transcribed genes [7]. For our study of the mouse genome we considered the methylation sensitive enzymes Asc I, Sac II, Not I and Hpa II as the mapping enzyme in combination with Nla III as the fragmenting enzyme. There are over a million restriction sites for Hpa II in the mouse genome and 38,684 for Sac II. Although both enzymes are excellent cutters, we concluded that the sequencing costs would be too high. We found only 2,500 restriction sites for Asc I and 6,012 sites for Not I with a respective average fragment size of 280,000 bp and $130,000 \mathrm{bp}$. We selected Not I since the recognition site contains two CG dinucleotides and approximately $62 \%$ of these sites are associated with a CPG island in the mouse genome. Though not all the genes are associated with a CpG Island it has been estimated that at least $40 \%$ of the annotated genes have a CpG Island in their corresponding promoter region.

For the generation of the mouse MSDK libraries we chose lung tissue from $\mathrm{C} 57 \mathrm{BL} / 6 \mathrm{~J}$ adult mice that were prenatally exposed to diets with increased (high methylation diet; HMD) or decreased (low methylation diet; LMD) methyldonor content and challenged with allergen in adulthood [9]. The mice gestated on both diets developed features characteristic of an allergic phenotype including airway hyper-reactivity, lung lavage eosinophilia, and increase serum IgE concentrations [9]. Genomic DNA was isolated from snap frozen lung tissue samples from two male mice for each group using a DNeasy kit (Qiagen, Valencia, CA) according to manufacturer instructions. Library construction was essentially performed as described for Digital Karyotyping with a few minor modifications [10]. The genomic DNA integrity was accessed by digestion with Sac I before use in library construction. For each library $2 \mu \mathrm{g}$ of genomic DNA was digested with the mapping enzyme Not I, ligated to biotinylated Not I linkers (Figure 1a) and digested with the fragmenting enzyme Nla III. DNA fragments containing the biotinylated linkers were isolated using streptavidin-coated magnetic beads (Dynal Biotech, Brown Deer, WI) and ligated to linkers including recognition sites for Mme I. The 21 bp sequence tags were released 


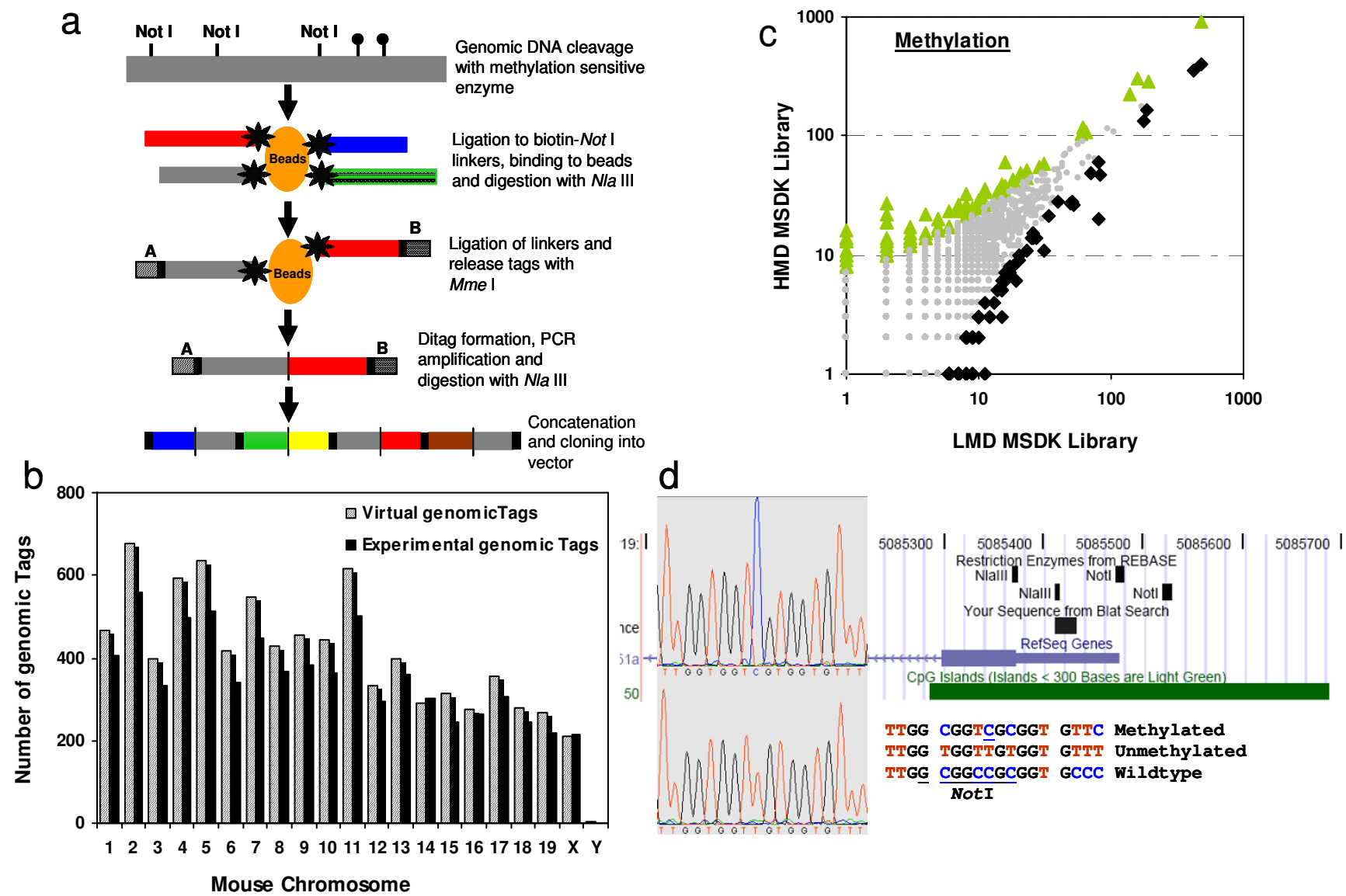

Figure I

a. Schematic view of the generation of a Not I MSDK library. The black lollipops indicate methylated Not I sites that will not be digested by the enzyme. Ib. The number of virtual tags (lined bars) per chromosome compared to the number of experimental tags (black bars) shows an unbiased sampling of the mouse genome. On average $>80 \%$ of experimental tags could be matched to a virtual tag after removal of all singletons from the pool of Not I MSDK genomic tags. Ic. A log 10 scale scatter plot comparison of genomic tag counts between the HMD ( $y$-axis) and the LMD ( $x$-axis) MSDK libraries. Significant differentially methylated genomic tags with a P-value $=0.05$ with higher counts in HMD are indicated by green triangles and those with higher counts in LMD are indicated by black diamonds. Points colored in grey are considered non-significant ( $\mathrm{P}$-value > 0.05). Id. Snapshot UCSC genome Browser displaying the location of the genomic tag found to be differentially methylated and associated with Tmem I 5 Ia on chromosome 19 (Table 2). The Not I restriction sites are also indicated. MSP was performed and the PCR products were sub cloned into a TOPO TA sequencing vector. Up to 12 independent clones with insert were selected for sequencing. The Sequence analysis of bisulfate modified genomic DNA from 2 HMD lung tissue samples revealed a methylated Not I site in the proximity of the gene encoding hypothetical protein Tmem I 5 Ia in 2 out of I 2 sequenced clones.

by digestion with Mme I as has been described for Long SAGE [11]. The isolated tags are self-ligated, PCR amplified, concatenated, cloned in pZero (Invitrogen, Carlsbad, CA), and sequenced. DNA from plasmid inserts containing serial genomic tags were purified and sequenced at Agencourt Bioscience Corporation (Beverly, MA). The SAGE 2000 software package enables the extraction of the genomic tags from the sequence files. Tag sequences, tag counts and gene associations were stored in a Microsoft
Access relational database for subsequent selection and matching to virtual tags.

We sequenced 66,758 and 69,498 tags for respectively the HMD and the LMD MSDK libraries. After removal of all singletons, we found 8,323 experimental genomic tags of which 6,310 were present in both libraries, 967 were specific for the HMD and 1046 for the LMD library. In order to match the experimental tags to their corresponding 
genomic loci, virtual genomic tags were extracted from the mouse genome sequence as obtained from Ensembl http:/ /www.ensembl.org using a python script http:// www.python.org. This identified a total of 10,577 virtual tags of which 8,414 are unique. Alignment of the experimental tags with the 10,577 virtual tags showed that $5,533(67 \%)$ could be matched to a unique position within the genome. A remaining 1,614 (19\%) matched to multiple regions in the genome and 1,176 (14\%) were not found. It is expected that the percentage of unmatched tags will decrease in time with the new releases of updated public mouse genome sequences. Comparison of the experimental tags with the virtual genomic tags per chromosome revealed an unbiased distribution of the experimental tags over the mouse genome (Figure 1b).

We identify differentially methylated loci in the mouse genome between the two libraries by applying the following selection criteria: mapping to a unique position to the mouse genome, and a fold difference in tag counts $\geq 5$. Furthermore, in order to find genomic tags with a significant difference in tag counts we used a z-score to quantify the strength of the observed difference in the proportions of individual tag sequences in the two libraries and generate a P-value. We considered a P-value $\leq 0.05$ as significant. Similar results were obtained when analyzing the data according to the sequence odds ratio and significance test http://cgap.nci.nih.gov/SAGE or the significance test available as part of the DiscoverySpace application [12]. In this way, we identified 82 genomic tags with a higher count in the LMD library and 71 genomic tags with higher counts in the HMD [see Additional files 1 and 2] representing differentially methylated loci within the mouse genome (Figure 1c). The MSDK method screens for unmethylated restriction enzyme sites in the genome therefore higher genomic tag frequencies correspond with a decreased level of methylation. This implies that the 82 genomic tags with increased counts in the LMD library are likely to show an increased level of methylation in the HMD library. Despite the limited sequence depth $(70,000$ genomic tags per library) we were able to find novel differentially methylated loci in our mouse model. Since most of the Not I sites are correlated with a CpG Island in the genome it can be expected that the differences in tag counts as identified by MSDK correlate with changes in transcription levels, which ultimately can influence the phenotype seen in our mouse model.

To validate the MSDK results we isolated total RNA from the same samples used in the MSDK analysis and performed Serial Analysis of Gene Expression (SAGE) profiling of these samples. This generated 102,868 and 68,778 transcript tags for respectively the HMD and LMD SAGE library. After normalization to correct for the differences in total tag counts in each SAGE library, we selected for transcripts with a fold-difference $\geq 5$ and a P-value $\leq 0.05$ as previously described [13]. Surprisingly we found 139 transcripts to be over expressed in the HMD SAGE library and about 1254 in the LMD SAGE library [see Additional files 3 and 4]. This is an almost 9-fold increase of transcription in the LMD sample (Figure 2a). Most likely this is due to a combination of increased DNA methylation in the HMD samples and a reduced amount of methyl donors in the LMD resulting in hypomethylation and poorly regulated transcription. It is important to note that not all methylation changes will affect transcriptional levels. Still, we could identify genes predicted to be methylated by MSDK analysis and with increased mRNA levels in the LMD SAGE library (Table 1). We also selected four genes from the list of differentially methylated loci for which the Not I site was located within a CpG Island in close proximity of the predicted transcription start site (based on the February 2006 Mouse Genome Assembly, http://genome.ucsc.edu/), and which were not expressed in sufficiently high levels to be significantly differentially expressed in the SAGE analysis (Table 2). Real-time PCR demonstrated an increase of expression level for Nfatc1, Jak2, Rcor3, Tmem151a, and the methyl-tranferases Dnmt1, Dnmt3A and Dnmt3B, in lung tissue from an independent set of animals prenatally exposed to a diet low in methyldonor content (LMD, Figure 2b). Similar results were obtained in lymphocyte-mononuclear cells isolated from spleen of HMD and LMD animals [9]. An increased expression of the methyl-transferases, especially those involve in de novo methylation (Dnmt3A and 3B), might partially account for the unexpected methylated genomic loci in the LMD library when compared to the HMD library [see Additional file 2] although other mechanisms of transcription regulation can not be excluded. Taken together the SAGE profiling and the real-time PCR confirmed the predicted changes in expression level by MSDK analysis.

A quantitative MSP assay was developed for parts of a CpG Island in close proximity of the genomic tags matched to Runx 3 and Nfatc1. Although this assay demonstrated an increased methylation level ( 10 to $50 \% \pm 20 \%$ ) in the genomic region analyzed in lung tissue and spleen, the results could be masked due to the use of a mixed cell population. Also the length of the CpG Island (over 1000 bp for Runx3) makes it difficult to design the assays. An extensive in vitro promoter analysis might be necessary to clearly identify the specific regulatory elements susceptible for methylation. Interestingly, the transcription factor Runx3 has been shown to be regulated by promoter methylation in human cancers $[14,15]$ and it was also shown that Runx 3 can be down regulated by an increase of Histone H3 methylation in human cancer cell lines [16]. More recently we have shown [9], that a demethylating agent can restore the transcription levels of Runx 3 in 

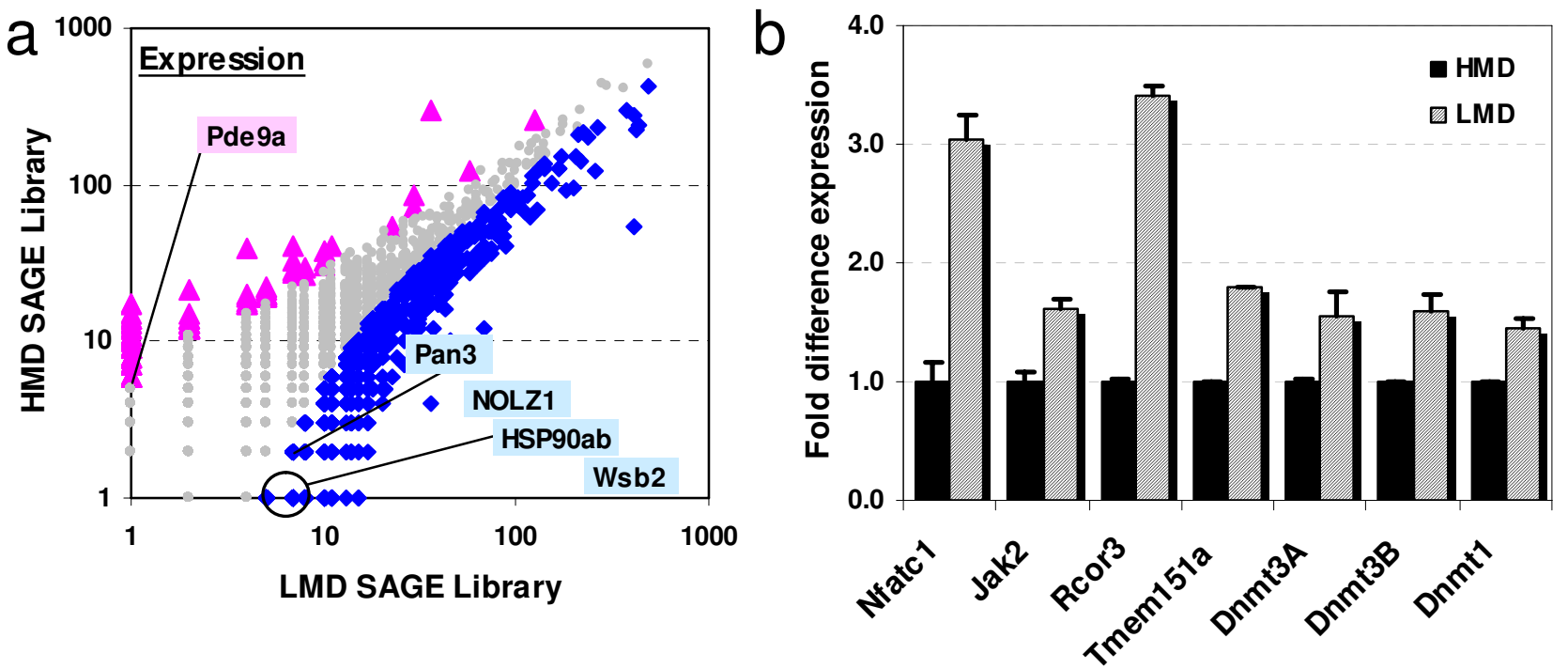

Figure 2

a. A log 10 scale scatter plot comparison of the transcript sequence tag counts as assessed by SAGE analysis between the HMD (y-axis) and the LMD (x-axis) libraries. Significant differentially expressed transcripts with a P-value $=0.05$ with higher expression in HMD are indicated by pink triangles and those with higher expression in LMD are indicated by blue triangles. Points colored in grey are considered non-significant ( $P$-value $>0.05)$. A few representative genes are indicated by their corresponding gene symbol. Pde9a, phosphodiesterase 9A; Nolzl, zinc-finger protein Nolz I; Pan3, Pan3 polyA specific ribonuclease subunit homolog; HSP90ab, heat shock protein $90 \mathrm{kDa}$ alpha, classBI; Wsb2, WD repeat and SOCS box-containing 2. 2b. Relative mRNA expression was measured by real-time PCR using SYBR-Green as described in Materials and Methods. Comparison between HMD/high responders $(n=6)$ and LMD/low responders $(n=6)$ in whole lung tissue obtained from challenged C57/BL6 mice. The values for the HMD samples were arbitrarily set to I in order to display the fold difference. Pvalues were calculated according to the Mann-Whitney U Test: Nfact I $=0.004 ;$ Jak2 $=0.077 ; \operatorname{Rcor} 3=0.002 ; \mathrm{Tmem} I 5 \mathrm{Ia}=$ 0.179; Dnmt3A $=0.05 \mathrm{I} ; \mathrm{Dnmt3B}=0.04 \mathrm{I}$; Dnmtl $=0.002$. All data are presented as the mean \pm standard error of the mean.

spleen cells from HMD animals, implying that the transcriptional changes are caused by methylation changes. The expression of nuclear factor of activated $\mathrm{T}$ cell 1 (Nfatc1) has also been described to be regulated by hyper- methylation in Hodgkin's lymphoma as well as by histone H3 acetylation and H3-K4 trimethylation [17]. It is not known whether Rcor3 (Rest corepressor 3) it self is regulated by methylation though depletion of Corest can

Table I: Comparison between MSDK and SAGE

\begin{tabular}{|c|c|c|c|c|c|c|c|}
\hline \multicolumn{3}{|c|}{ Methylation Specific Digital Karyotyping } & \multicolumn{2}{|c|}{ Gene Information } & \multicolumn{3}{|c|}{ Serial Analysis of Gene Expression } \\
\hline $\begin{array}{l}\text { MSDK Genomic Tag } \\
\text { Sequence }\end{array}$ & MSDK LMD & MSDKHMD & Gene Symbol & Gene Description & SAGE Tag Sequence & SAGE LMD & SAGE HMD \\
\hline $\begin{array}{c}\text { ACCTACCCAGGCA } \\
\text { GCCT }\end{array}$ & 8 & 0 & Zfp503 & $\begin{array}{l}\text { zinc-finger protein } \\
\text { NOLZI }\end{array}$ & TTTGTTACAA & 5 & 0 \\
\hline $\begin{array}{c}\text { GCAGCGTCCCGGG } \\
\text { TCGG }\end{array}$ & 6 & 0 & Hsp90ab I & $\begin{array}{l}\text { heat shock protein } 90 \\
\mathrm{kDa} \text { alpha, } \mathrm{BI}\end{array}$ & GTGAGCCCAT & 4 & 0 \\
\hline $\begin{array}{c}\text { AACAGTGGCGGCG } \\
\text { GCGG }\end{array}$ & 5 & 0 & Pan3 & $\begin{array}{l}\text { PAN3 polyA specific } \\
\text { ribonuclease subunit }\end{array}$ & TCGCGTCGCT & 7 & 2 \\
\hline $\begin{array}{c}\text { GCGCAGGCGACCC } \\
\text { GGGG }\end{array}$ & 6 & 1 & Wsb2 & $\begin{array}{l}\text { WD repeat and SOCS } \\
\text { box-containing } 2\end{array}$ & GGATGTACCC & 5 & 1 \\
\hline $\begin{array}{c}\text { AACAAAAAGGGTCT } \\
\text { GTG }\end{array}$ & 2 & 10 & Pde9a & phosphodiesterase 9A & TAAATTCCAC & 0 & 6 \\
\hline
\end{tabular}

Genomic tags are indicated as 17 bp sequences with the corresponding counts as found in each MSDK library. Transcript tags are indicated as 10 bp sequences with the corresponding expression level as found in each SAGE library. Genomic DNA and RNA for MSDK and SAGE analysis was isolated from lung tissue from C57BL/6] adult mice that were prenatally exposed to nutritional changes as described in [9]. 
Table 2: Selected candidate genes for real-time PCR analysis

\begin{tabular}{ccccccl}
\hline Genomic Tag & LMD & HMD & Chr & Position & Strand & Gene \\
\hline CGACAGAGGGCCGGGGG & 6 & 0 & 18 & 80870439 & - & Nfatcl (nuclear factor of activated T-cells) \\
CAGAATGGGTGCTGCCT & 6 & 0 & 19 & 29317014 & + & Jak2 (Janus kinase 2) \\
GTAGAGGAGGGGGAGAG & 8 & 0 & 1 & 193838624 & + & Rcor3 (REST corepressor 3) \\
ATAAGCAGGGGTGCGGG & 8 & 0 & 19 & 5085417 & + & Tmem I5la (Hypothetical protein LOC38II99) \\
\hline
\end{tabular}

Genomic tags are indicated as 17 bp sequences with the corresponding counts as found in each MSDK library, location within the genome, strand orientation and associated gene. A complete list can be found in Additional file I and [9].

result in derepression of REST responsive gene expression and increased methylation of Histone H3-K4 [18,19]. Other examples of genes regulated by methylation that have been identified in our study as differentially methylated candidate genes [see Additional files 1 and 2] can be found in the literature like Cited4, which is epigenetically silenced in the vast majority of human oligodendroglial tumors [20]. Taken together these findings indicate that it is very likely that our list of differentially methylated loci is actually regulated by DNA methylation but it also indicates that the contribution of other epigenetic mechanisms like histone modifications affecting the accessibility of chromatin can not be excluded.

\section{Differential gene expression and functional analysis}

We applied the Ingenuity Pathway Analysis program to explore the list of differentially expressed genes. The top canonical pathways that are significantly associated with the differentially expressed genes in the lungs of HMD or LMD animals are represented in Figure 3. The significance is determined by a high ratio (or percentage of genes in pathway found in the gene list) and by a high negative logarithm of the P-value; indicating that the pathway is significantly associated with the data and that a large portion of the corresponding canonical pathway may be affected. Interestingly, we see an association of the folate biosynthesis pathway in the HMD animals. This pathway plays a role in the synthesis of S-adenosyl methione, the main methyl donor group in the process of DNA methylation [21] implying an association between the maternal nutritional intake and increase of DNA methylation in this group. Dietary changes can clearly have an effect on the transcriptional profiles thereby adding another variably that can affect the phenotypic responses to exposure.

\section{Conclusion}

Perinatal dietary changes in methyl donor content can lead to alterations in expression profiles as well as subtle changes in DNA methylation. Using MSDK, a method that allows a comprehensive and unbiased genome-wide methylation profiling, we uncovered differentially methylated loci in the progeny of mice maternally subjected to diets high and low in methyl donors. The depth and coverage of the approach is dependent on the restriction enzyme used and can be adjusted as needed. The MSDK technology is sequence based, quantitative and unbiased without the requirement of special equipment other than a DNA sequencer. An increase in throughput and large reduction of the sequencing costs is expected with the recent development of multiplex paired-end ditag sequencing technologies [22,23]. Most importantly; the data is digitally archived, relative easy to analyze and independent of an arbitrarily chosen reference. MSDK is applicable for mouse models in a mixed cell population, and may be a useful approach to determine whether environmental exposures can lead to epigenetic changes in complex heritable human diseases.

\section{Methods}

\section{Primers and linkers}

Not-MSDK linkers: 5'-/5Phos/GGCCGCACCCAACTCGATTACGAACCTCTGC-3' and 5'-/5Bio/TTTGCAGAGGTTCGTAATCGAGTTGGGTGC-3'. These linkers were obtained PAGE purified and modified from Integrated DNA Technologies, Coralville, IA.

RT-PCR primers: Jak2 Forward 5'-TCTGTGGGAGATCTGCAGTG-3', Jak2 Reverse 5'-CACGGATGACAGCTCTGAAA-3'; Nfatc1 5orward 5'TCATCCTGTCCAACACCAAA-3', Nfatc1 Reverse 5'TCACCCTGGTGTTCTTCCTC-3'; Rcor3 Forward 5'-CATGGATGGAAACGACAGTG-3', Rcor3 Reverse 5'-AGTTGCCTCAGGATGGTGTT-3'; B2M Forward 5'ATTCACCCCCACTGAGACTG-3', B2M Reverse 5'GCTATTTCTTTCTGCGTGCAT-3'; PGK1 Forward 5'CAAGGCTTTGGAGAGTCCAG-3', PGK1 Reverse 5'-TGTGCCAATCTCCATGTTGT-3'; Actb Forward 5'-TCCGTAAAGACCTCTATGCC-3'; Actb Reverse 5'TACTCCTGCTTGCTGATCC-3'

Tmem151a Forward 5'-AGGGCGAAGGTGGAGACT-3', Tmem151 Reverse 5'-GGCATGGATGAGCAGTGTAA-3'; mDnmt3a Forward 5'-TGACGCCAAAGAAGTGTCTG-3, mDnmt3a Reverse 5'-TTCAGTGCACCACAGGATGT-3'; mDnmt3b Forward 5'-ACTTGGTGATTGGTGGAAGC-3', mDnmt3b Reverse 5'-CCAGAAGAATGGACGGTTGT-3'; mDnmt1 Forward 5'-TCCTCAGGGACCATATCTGC-3', mDnmt1 Reverse 5'-CTGCACAGGAACAGACTCCA-3'. MSP primers: Runx3 methylated forward 5'AGAAAATCGTTTTGGGTGTTATC-3', reverse 5'-AATTTC- 


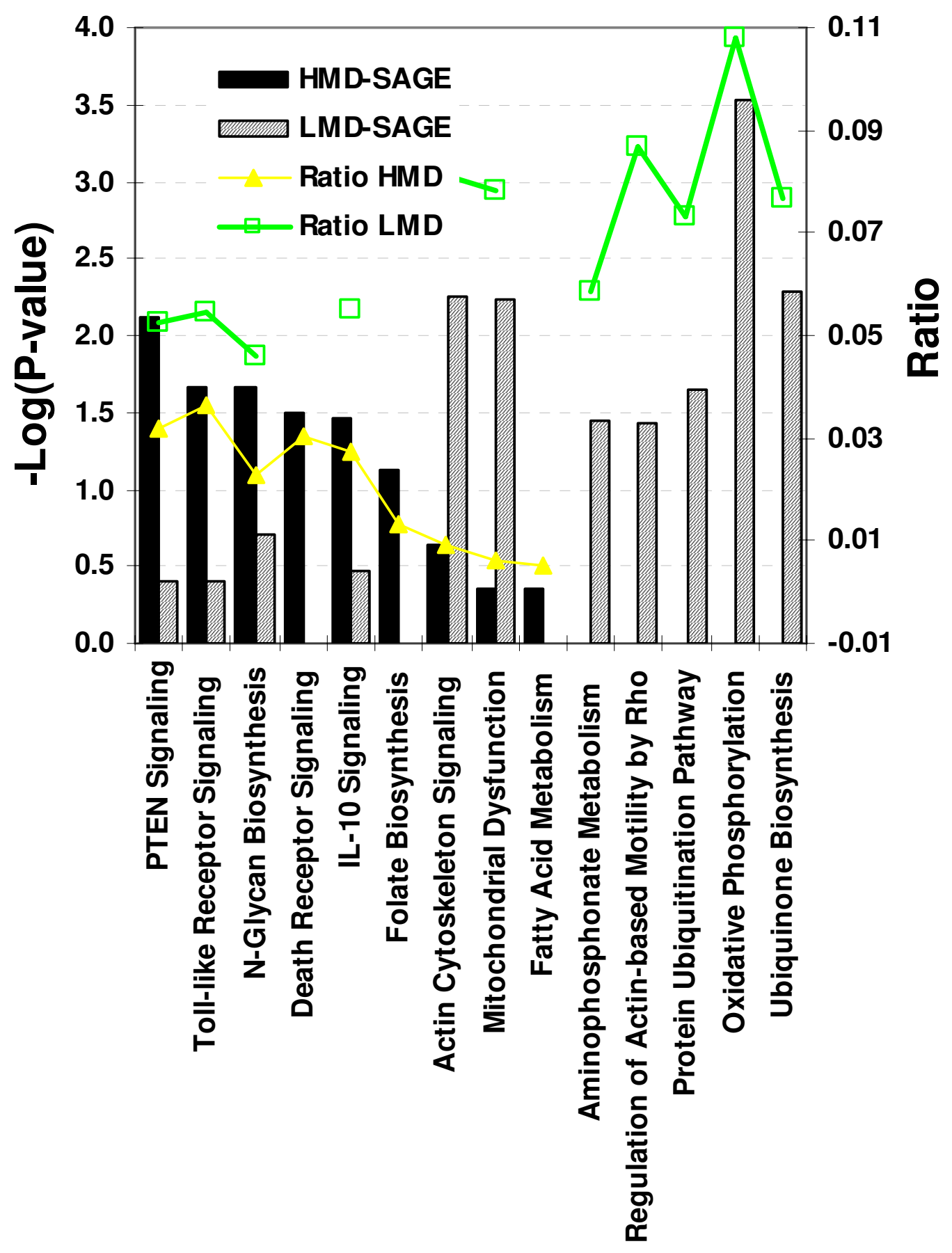

Figure 3

Pathway analysis. Most significant canonical pathways associated with the HMD and LMD SAGE expression profiles according to the IPA pathway analysis tool. The significance of the association between the dataset and the canonical pathway was measured as a ratio (number of genes from the dataset that map to the pathway divided by the total number of molecules that exist in the canonical pathway). A Fischer's Exact Test was used to calculate a P-value. 
CAACCTCCTAACTACGAC-3'; Nfatc1 methylated forward 5'-GTAGTTTAGTTAGGGAGGAGGATTC-3', reverse 5'TACGAAAACGAAAAAACTTTACGAC-3'; Actb methylated forward 5'-TGTGATTGATAGTAGGAAGGTGTGA-3'; reverse 5'-ACCCAAATCCAAAAATCACG-3'.

\section{SAGE library construction and statistical analysis of tag counts differences}

SAGE libraries from the same samples used for MSDK analysis were constructed using Nla III as the anchoring enzyme and $B s m F$ I as the tagging enzyme according to a micro-SAGE protocol [13]. The SAGE library clones were arrayed and inserts were purified and sequenced at Agencourt Bioscience Corporation. The SAGE 2000 software version 4.12 (available at http://www.sagenet.org) was used to extract SAGE tags from the original sequence files, remove duplicate ditags, remove linker sequences, remove one base pair variations of linker sequences and tabulate the occurrence of each tag. Tag sequences, tag counts and gene associations were stored in a Microsoft Access relational database for subsequent analysis. P-values for differentially expressed transcripts were calculated according to the sequence odds ratio and significant test http:// cgap.nci.nih.gov/SAGE). Similar results were obtained when using the SAGE software Monte Carlo approach or the significance test available as part of the DiscoverySpace application [12]. The generated mouse SAGE libraries have been deposit at the Geo Website (GSE11676).

\section{Gene Ontology and Canonical Pathway analysis}

Ingenuity Pathway Analysis (IPA, Ingenuity Systems ${ }^{\circledR}$, http://www.ingenuity.com) is a web-based application that enables the visualization, discovery and analysis of molecular interaction networks within gene expression profiles. The generated SAGE datasets and the corresponding expression levels, represented as the $\log _{2}$ ratios, were uploaded within the IPA database for further analysis. Both gene symbols and gene bank accession numbers were used with no apparent differences in results. These genes, called focus genes, were overlaid onto a global molecular network developed from information contained in the Ingenuity knowledge base. The IPA knowledge base represents a proprietary ontology of over 600,000 classes of biologic objects spanning genes, proteins, cells and cell components, anatomy, molecular and cellular processes, and small molecules. Networks of the focus genes were then algorithmically generated based on their connectivity. The Functional Analysis of a network identified the biological functions and/or diseases that were most significant to the genes in the network. The network genes associated with biological functions and/or diseases in the Ingenuity knowledge base were considered for the analysis. Fischer's exact test was used to calculate a P-value determining the probability that each biological function and/or disease assigned to that network is due to chance alone. Canonical Pathways Analysis identified the pathways from the Ingenuity Pathways Analysis library of canonical pathways that were most significant to the dataset. The significance of the association between the dataset and the canonical pathway was measured in 2 ways: 1) a ratio of the number of genes from the dataset that map to the pathway divided by the total number of molecules that exist in the canonical pathway is displayed. 2) Fischer's Exact Test was used to calculate a P-value.

\section{RNA isolation and real-time PCR}

Total RNA was extracted from frozen lung tissue using the RNAgents total RNA isolation system (Promega, Madison, WI, USA). Equal amounts of total RNA (5 $\mu \mathrm{g})$, as determined by the Ribo-Green RNA Quantification kit (Molecular Probes, Eugene, OR, USA), were used in a $20 \mu \mathrm{l} \mathrm{cDNA}$ synthesis reaction primed with oligo-dT (Superscript II; Invitrogen, Carlsbad, CA, USA). Control reactions were prepared in parallel without reverse transcriptase. Prior to cDNA synthesis, residual genomic DNA was removed from total RNA with a DNase I treatment (DNA-free; Ambion, Austin, TX, USA). Quantitative PCR was performed with a 7900TH Fast Real-Time PCR system (Applied Biosystems, Foster City, CA, USA) using SYBRGreen. PCR reactions were performed in triplicate, and the threshold cycle numbers were averaged. Gene expression levels were normalized to three genes: ACTB (actin, beta), B2M (beta-2-microglobulin and PGK1 (phosphoglycerate kinase 1). The relative expression levels were calculated in comparison to the levels in total RNA from naïve mouse brain (Ambion, Austin, TX) according to the Comparative $\mathrm{C}_{\mathrm{t}}$ method in which the relative expression equals $2^{-\Delta \Delta \mathrm{Ct}}$. PCR primers were designed using the Primer 3 interface http://frodo.wi.mit.edu/cgi-bin/primer3/

primer3 www.cgi. Data is presented as the mean \pm standard error of the mean. Significant differences between groups were identified by analysis of variance. Individual comparisons between groups were confirmed by the twotailed Student's t test or the Mann-Whitney U Test. A Pvalue $\leq$ to 0.05 was considered significant.

\section{Methylation Specific PCR}

Sodium bisulfite modification of 500 ng genomic mouse lung DNA was performed using the EZ DNA MethylationGold Kit (Zymo Research, Orange, CA) according to manufacture's instructions. Modified DNA was eluted from the column with 20 ul elution buffer, aliquoted and stored at $-20^{\circ} \mathrm{C}$. PCR reactions were performed essentially as described using 2 ul modified genomic DNA [24]. CpG islands at the $5^{\prime}$ region and up to the first exon of each gene were identified using online tools http:// genome.ucsc.edu/. The corresponding sequences were downloaded for primer design using MethPrimer [25] and Primer3 http://frodo.wi.mit.edu/cgi-bin/primer3/ 
primer3 www.cgi. Primers were designed to contain at least one $\mathrm{CpG}$ dinucleotide. Amplified products were analyzed by gel electrophoresis. Data was represented as mean \pm standard error of the mean. Significant differences between groups were identified by analysis of variance. Individual comparisons between groups were confirmed by the two-tailed Student's t test or the Mann-Whitney U test. A P-value $=$ to 0.05 was considered significant.

\section{TopoA cloning and sequencing}

Amplified MSP products were analyzed by gel electrophoresis prior to further processing and purified by phenol extraction and ethanol precipitation. Approximately $70 \mathrm{ng}$ of PCR product was used in a ligation reaction according to manufactures instructions using a TOPO TA cloning kit for Sequencing (Invitrogen, Carlsbad, CA). Transformants were analyzed by colony PCR with universal M13 primers. These PCR products were subsequently purified using a QIAquick PCR Purification Kit (Qiagen USA) and eluded from the columns in a $30 \mathrm{ul}$ volume. For the sequencing reaction $4 \mathrm{ul}$ purified PCR product with 5 pmole T7 universal primer was used in combination with a Dynamic ET Terminator Cycle Sequencing Kit (Amersham Biosciences, Piscataway, NJ). Post-sequencing cleanup was performed according to manufactures instructions.

\section{Authors' contributions}

KB designed, performed computational analyses and generated most of experimental data. NWB generated experimental data. JKT provided bioinformatics and statistical support. SM provided samples. SM, JHW, SG, DMB and $\mathrm{ZL}$ all contributed in the collection of mouse tissue samples. $\mathrm{KB}, \mathrm{JWH}$ and DAS contributed to the overall design of this project, provided critique of the results, and participated in manuscript conceptual development and editing. All authors read and approved the final manuscript.

\section{Additional material}

\section{Additional file 1}

Differentially methylated genomic tags and nearest genes. Files 1 and 2 catalogs respectively the 82 genomic sequence tags with higher counts in the LMD library. Also indicated are the nearest genes.

Click here for file

[http://www.biomedcentral.com/content/supplementary/14712164-9-598-S1.doc]

\section{Additional file 2}

Differentially methylated genomic tags and nearest genes. Catalogs the 71 genomic sequence tags with higher counts in the HMD MSDK library representing differentially methylated loci within the mouse genome. Also indicated are the nearest genes.

Click here for file

[http://www.biomedcentral.com/content/supplementary/14712164-9-598-S2.doc]

\section{Additional file 3}

Differentially expressed SAGE transcripts. Represents the transcript tags significantly over expressed in the HMD SAGE library.

Click here for file

[http://www.biomedcentral.com/content/supplementary/1471-

2164-9-598-S3.doc]

\section{Additional file 4}

Differentially expressed SAGE transcripts. Represents the transcript tags significantly over expressed in the LMD SAGE library.

Click here for file

[http://www.biomedcentral.com/content/supplementary/1471-

2164-9-598-S4.doc]

\section{Acknowledgements}

This work was supported by NIEHS and NHLBI Intramural Research Programs (DAS) and HL09I335 (JWH).

\section{References}

I. Esteller M: Cancer epigenomics: DNA methylomes and histone-modification maps. Nat Rev Genet 2007, 8(4):286-298.

2. Robertson KD: DNA methylation and human disease. Nat Rev Genet 2005, 6(8):597-610.

3. Waterland RA, Michels KB: Epigenetic Epidemiology of the Developmental Origins Hypothesis. Annual Review of Nutrition 2007, 27(I):363-388.

4. Jirtle RL, Skinner MK: Environmental epigenomics and disease susceptibility. Nat Rev Genet 2007, 8(4):253-262.

5. Waterland RA, Jirtle RL: Early nutrition, epigenetic changes at transposons and imprinted genes, and enhanced susceptibility to adult chronic diseases. Nutrition 2004, 20(1):63-68.

6. Zilberman D, Henikoff $S$ : Genome-wide analysis of DNA methylation patterns. Development 2007, I34(22):3959-3965.

7. Hu M, Yao J, Cai L, Bachman KE, Brule $F$ van den, Velculescu V, Polyak $\mathrm{K}$ : Distinct epigenetic changes in the stromal cells of breast cancers. Nat Genet 2005, 37(8):899-905.

8. Hu M, Yao J, Polyak K: Methylation-specific digital karyotyping. Nat Protocols 2006, I(3): |62|-1636.

9. Hollingsworth J, Maruoka S, Boon K, Garantziotis S, Li Z, Tomfohr J, Bailey N, Potts E, Whitehead G, Brass D, Schwartz DA: In utero supplementation with methyl-donors enhances allergic airway disease in mice. Journal of Clinical Investigation 2008, I I 8( I 0):3462-3469.

10. Wang T, Maierhofer C, Speicher MR, Lengauer C, Vogelstein B, Kinzler KW, Velculescu VE: Digital karyotyping. PNAS 2002, 99(25): $16|56-16| 6 \mid$.

II. Saha S, Sparks AB, Rago C, Akmaev V, Wang CJ, Vogelstein B, Kinzler $\mathrm{KW}$, Velculescu VE: Using the transcriptome to annotate the genome. Nat Biotech 2002, 20(5):508-5 12

12. Robertson N, Mehrdad Oveisi-Fordorei M, Zuyderduyn SD, Richard J, Varhol RJ, Fjell C, Marra M, Jones S, Siddiqui A: DiscoverySpace: an interactive data analysis application. Genome Biology 2007, 8(I):R6-RI3.

13. Boon K, Edwards J, Siu I, Olschner D, Eberhart C, Marra M, Strausberg R, Riggins G: Comparison of medulloblastoma and normal neural transcriptomes identifies a restricted set of activated genes. Oncogene 2003, 22(48):7687-7694.

14. Mueller W, Nutt CL, Ehrich M, Riemenschneider MJ, von Deimling A, Boom D van den, Louis DN: Downregulation of RUNX3 and TES by hypermethylation in glioblastoma. Oncogene 2006, 26(4):583-593.

15. Subramaniam M, Chan J, Soong R, Ito K, Ito $Y$, Yeoh K, Salto-Tellez $M$, Putti T: RUNX3 inactivation by frequent promoter hypermethylation and protein mislocalization constitute an early event in breast cancer progression. Breast Cancer Res Treat 2009, I I3(I):II3-12I. 
16. Fujii S, Ito K, Ito $\mathrm{Y}$, Ochiai A: Enhancer of Zeste Homologue 2 (EZH2) Down-regulates RUNX3 by Increasing Histone H3 Methylation. J Biol Chem 2008, 283(25): I7324-17332.

17. Akimzhanov A, Krenacs L, Schlegel T, Klein-Hessling S, Bagdi E, Stelkovics E, Kondo E, Chuvpilo S, Wilke P, Avots A, Gattenlohner S, Muller-Hermelink H, Palmetshofer A, Serfling E: Epigenetic Changes and Suppression of the Nuclear Factor of Activated T Cell I (NFATCI) Promoter in Human Lymphomas with Defects in Immunoreceptor Signaling. Am J Pathol 2008, I72(1):215-224.

18. Lee MG, Wynder C, Cooch N, Shiekhattar R: An essential role for CoREST in nucleosomal histone 3 lysine 4 demethylation. Nature 2005, 437(7057):432-435.

19. Lakowski B, Roelens I, Jacob S: CoREST-like complexes regulate chromatin modification and neuronal gene expression. Journal of Molecular Neuroscience 2006, 29(3):227-239.

20. Tews B, Roerig P, Hartmann C, Hahn M, Felsberg J, Blaschke B, Sabel M, Kunitz A, Toedt G, Neben K, Benner A, Deimling A, von Reifenberger G, Lichter P: Hypermethylation and transcriptional downregulation of the CITED4 gene at Ip34.2 in oligodendroglial tumours with allelic losses on Ip and I9q. Oncogene 2007, 26(34):5010-5016.

21. Waterland RA: Assessing the Effects of High Methionine Intake on DNA Methylation. J Nutr 2006, 136(6): 1706S-17I0.

22. $\mathrm{Ng}$ PWC, Ruan Y: Paired-end diTagging for transcriptome and genome analysis. Volume 2007. John Wiley \& Sons, Inc; 2007.

23. Nielsen K, Hogh AL, Emmersen J: DeepSAGE - digital transcriptomics with high sensitivity, simple experimental protocol and multiplexing of samples. Nucl Acids Res 2006, 34(19):e I 33.

24. Herman JG, Graff JR, Myohanen S, Nelkin BD, Baylin SB: Methylation-specific PCR: A novel PCR assay for methylation status of CpG islands. PNAS 1996, 93(18):982I-9826.

25. Li L-C, Dahiya R: MethPrimer: designing primers for methylation PCRs. Bioinformatics 2002, I8(II):|427-|43|.

Publish with Bio Med Central and every scientist can read your work free of charge

"BioMed Central will be the most significant development for disseminating the results of biomedical research in our lifetime. "

Sir Paul Nurse, Cancer Research UK

Your research papers will be:

- available free of charge to the entire biomedical community

- peer reviewed and published immediately upon acceptance

- cited in PubMed and archived on PubMed Central

- yours - you keep the copyright
BioMedcentral 